\title{
Approach to inter-satellite time synchronization for micro-satellite cluster
}

\author{
XU Jiuling, ZHANG Chaojie*, WANG Chunhui, and JIN Xiaojun
}

Micro-Satellite Research Center, Zhejiang University, Hangzhou 310027, China

\begin{abstract}
Micro-satellite cluster enables a whole new class of missions for communications, remote sensing, and scientific research for both civilian and military purposes. Synchronizing the time of the satellites in a cluster is important for both cluster sensing capabilities and its autonomous operating. However, the existing time synchronization methods are not suitable for microsatellite cluster, because it requires too many human interventions and occupies too much ground control resource. Although, data post-process may realize the equivalent time synchronization, it requires processing time and powerful computing ability on the ground, which cannot be implemented by cluster itself. In order to autonomously establish and maintain the time benchmark in a cluster, we propose a compact time difference compensation system (TDCS), which is a kind of time control loop that dynamically adjusts the satellite reference frequency according to the time difference. Consequently, the time synchronization in the cluster can be autonomously achieved on-orbit by synchronizing the clock of other satellites to a chosen one's. The experimental result shows that the standard deviation of time synchronization is about $102 \mathrm{ps}$ when the carrier to noise ratio $(\mathrm{CNR})$ is $95 \mathrm{dBHz}$, and the standard deviation of corresponding frequency difference is approximately $0.36 \mathrm{~Hz}$.
\end{abstract}

Keywords: time difference measurement, time synchronization, dynamical reference frequency adjustment, micro-satellite cluster.

DOI: $10.21629 / J S E E .2018 .04 .15$

\section{Introduction}

Compared with the traditional monolithic satellite with tailored design and long develop time, the micro-satellites (satellites with lower weight and smaller size) are becoming more attractive due to lower development costs and shorter lead time [1]. The major drawback of these onetime use traditional satellites is that they limit the possible adaptations of satellites to the likely changes in their

Manuscript received June 29, 2017.

*Corresponding author.

This work was supported by the National Natural Science Foundation of China (61401389) and the Joint Fund of the Ministry of Education of China (6141A02033310). requirements or environment during their life cycle [2]. Meanwhile, the miniaturization achieved by utilizing commercial-off-the-shelf (COTS) component spurs the development of microsatellites [3], such as QB50 mission [4]. Consequently, transforming a traditional monolithic satellite into multiple micro-satellites to establish an on-orbit cluster is a cost-effective method to meet the demand of various missions [5].

The concept of satellite cluster was originally derived from distributed space system, which was first introduced to astronomical and planetary applications, but later such approaches also were proposed for earth observation [6]. Generally, the satellites in a cluster are intentionally placed close together to increase the sensing capabilities or extend the coverage [7]. The reliable inter-satellite communication system in large-delayed environment $[8,9]$ and its multiple access protocol [10] are the basic technology for the cluster. Besides, the advanced systems, such as accurate inter-satellite ranging system, the precise attitude determination and the control systems, are supposed to be used in high precision scenarios to avoid satellite collisions and maintain the cluster configuration. Some missions relying on two cooperative satellites, such as gravity recovery and climate experiment (GRACE) (amid at gravimetry [11] and climate monitoring [12]), CanX-4\&5 [13] (technologies validation) project for on-board autonomy-3 (PROBA-3) (target formation maintenance [14] and precise formation flying demonstration [15]), prototype research instruments and space mission advancement (PRISMA) [16] (new technology testing), and Terra SAR-X add-on for digital elevation measurement (Tandem-X) [17] (synthetic aperture radar interferometer), have already been successfully accomplished. Another advantage of using distributed satellite system for earth observation is that it can provide flexible baseline to achieve bi-/multi-static synthetic aperture radar (SAR) [18].

In order to enhance the cooperative sensing capability of cluster, the measurement data from various satellites 
should be carefully marked with a unified timestamp, and the states synchronization of the satellites is also important for both cluster establishment and cluster keeping [19]. Additionally, future space missions are envisioned to become more complex and operate farther from the Earth, and will need to support autonomous operations with minimal human intervention [20]. Therefore, the establishment of a well autonomous operating micro-satellite cluster with a unified time benchmark is fundamentally important.

Generally speaking, the existing and emerging time synchronization (time sync for short) can be categorized either as time correction or as data post-process. The former is the correction of satellites clock with respect to a certain time base, such as GPS system time. First, each GPS satellite carries multiple atomic frequency standards (AFS) to provide a stable reference frequency for satellite clock. Second, the dedicated control station commands the satellite AFS, monitors their performance, and maintains estimates of satellite clock bias, drift, and drift rate to support the generation of clock corrections for the unification of GPS system time [21]. However, the clock errors for a typical satellite are on the order of $0.8 \mathrm{~m}$ (2.6 ns) [22]. The latter has been adopted by GRACE [23] and gravity recovery and interior laboratory (GRAIL) [24,25] to accomplish microlevel inter-satellite measurement links, and the measurement accuracy of time difference between two satellites is about 150 ps [26]. By exchanging $\mathrm{K}$ and Ka carrier signals, measuring the time difference between two orbiting clocks, and downloading measurement information, GRACE and GRAIL have been achieving a quantum leap in the knowledge of the Earth's and the moon's gravitational field, respectively [26].

Although, AFS can provide highly stable reference frequency, it is space-occupying, high-cost and powerconsuming for microsatellites. Additionally, it should be considered that the frequency standard of the ordinary oscillator, i.e., the temperature compensate crystal oscillator (TCXO) on micro-satellite is not as stable and accurate as AFS. The oscillator performance deterioration caused by unpredictable effects of space environment might lead to the disruption of time unification within several minutes, which is too difficult to be estimated and corrected by the limited number of dedicated ground stations. The post-process may help to reduce the influence on time difference due to the powerful computing capability on the ground, but it requires more human intervention, which is contrary to the goal of establishing an autonomous microsatellite cluster. Besides, taking GRAIL for example, it is a very complicated work to synchronize time, since there are four independent clocks to be synchronized during post-process [27].
In order to achieve the purpose of autonomous time sync for microsatellite cluster, we propose a compact time difference compensation system (TDCS), which is completely different from the existing approaches, because it can provide the ability to on-orbit autonomous time sync. In terms of TDCS, the time sync is achieved by dynamically adjusting the reference frequency of satellite according to the current time difference, and time difference will be compensated and kept near zero at last. The current time difference is usually measured by the methods of two-way satellite time and frequency transfer (TWSTFT) [28,29], or GPS-CV [30,31]. However, in this paper, we adopt a kind of TWSTFT architecture-based time difference measurement system (TDMS), which computes the time difference by measuring the phase difference of the pseudonoise (PN) code of the transmitter between different satellites, since the phase of the PN code and the satellite clock are driven by the same on-board frequency standard.

As a result, in a cluster, the time of other satellites (the followers) will be synchronized to the time of a certain satellite (the leader), so the followers have the same time benchmark as the leader. In other words, the time benchmark of the cluster is the time benchmark of the leader. More importantly, the time benchmark in the cluster is not maintained by atomic frequency standard but kept by dynamically synchronizing the time of the followers to that of the leader. Consequently, the TDCS does not ask for a high-precision and high-stability frequency standard (atomic frequency standard), but an oscillator, TCXO or oven controlled crystal oscillator (OCXO) with good performance, can also serve as a qualified reference frequency standard. This is very meaningful to the low-cost, spacelimited and power-constrained micro-satellite applications. However, this kind of time benchmark only exists in that cluster, which may not synchronize to the global time standard such as universal time coordinated (UTC) or greenwich mean time (GMT).

The remainder of the paper is organized as follows. The system introduction and error analysis are discussed in Section 2. Simulation and experiment results are presented in Section 3 and Section 4, and this paper is concluded in Section 5.

\section{System overview}

\subsection{Overview of time sync system}

Taking two satellites for example, as shown in Fig. 1, the satellite equipped with TDCS is designated as the follower, and the other one serves as the leader. The clock of the leader is driven by the onboard oscillator (OSC) directly, while the clock of the follower is derived from the refer- 
ence frequency $f_{O S C}^{\prime}$, which is determined by the current time difference provided by TDMS. The follower has two working states: in the open loop state, TDCS is disabled, so that the time difference is time-varying; in the closed loop state, TDCS dynamically adjusts $f_{O S C}^{\prime}$ to ensure that time difference will be converged to zero.

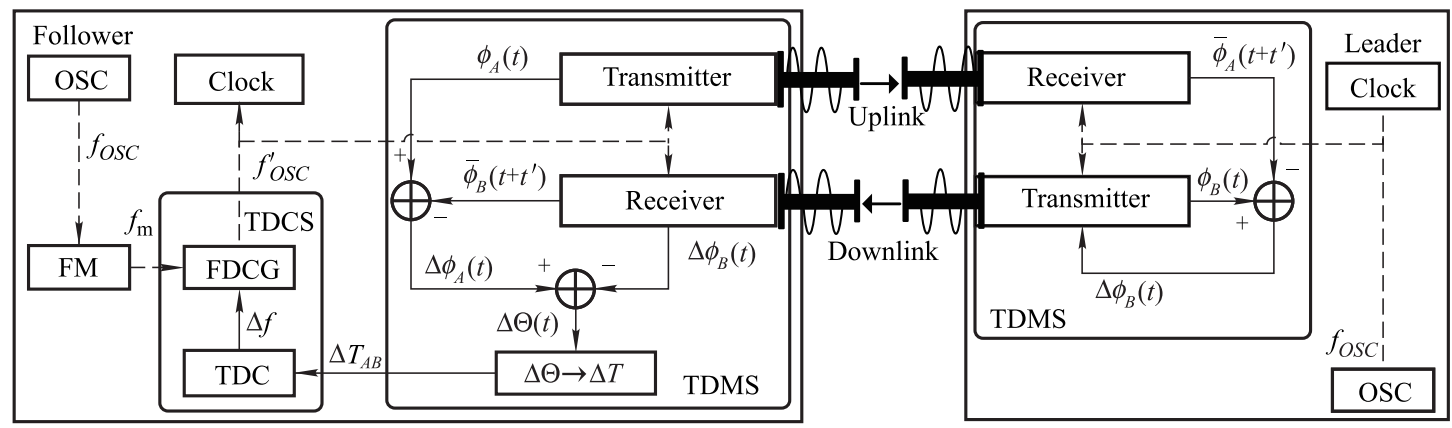

Fig. 1 System overview

\subsection{TDMS model}

The TDMS is based on a direct-sequence spectrum spread (DSSS) transponder, we use PN code phase to measure time difference indirectly. In Fig. 1, both $\phi_{x}$ and $\bar{\phi}_{x}$ mean that the signal is generated by the satellite $x$ ( $x$ can be A or B which represents the follower and the leader, respectively). $\phi_{x}(t)$ is the PN code phase transmitted by satellite $x$ at moment $t . \bar{\phi}_{x}\left(t+t^{\prime}\right)$ is the $\mathrm{PN}$ code phase received by the other one at moment $t+t^{\prime}$. The observations of TDMS are the phase difference of transmitted PN code and received PN code, denoted as $\Delta \phi_{A}(t)$ and $\Delta \phi_{B}(t)$.

$$
\begin{aligned}
& \Delta \phi_{A}(t)=\phi_{A}(t)-\bar{\phi}_{B}\left(t+t^{\prime}\right) \\
& \Delta \phi_{B}(t)=\phi_{B}(t)-\bar{\phi}_{A}\left(t+t^{\prime}\right)
\end{aligned}
$$

Because the wireless links are symmetrical, the propagation delays of uplink/downlink are equal in value, both delays can be written as $t^{\prime}$ [28]. The leader sends $\Delta \phi_{B}(t)$ to the follower through downlink channel. Therefore, the difference of these observations $\Theta(t)$ can be computed by the follower.

$$
\begin{gathered}
\Theta(t)=\Delta \phi_{A}(t)-\Delta \phi_{B}(t)= \\
\phi_{A}(t)-\bar{\phi}_{B}\left(t+t^{\prime}\right)-\phi_{B}(t)+\bar{\phi}_{A}\left(t+t^{\prime}\right)
\end{gathered}
$$

The received PN code phase at a certain moment can be replaced with the PN code phase at the transmitting moment,

$$
\begin{aligned}
& \bar{\phi}_{A}\left(t+t^{\prime}\right)=\phi_{A}(t)-2 \pi f_{P N} t^{\prime} \\
& \bar{\phi}_{B}\left(t+t^{\prime}\right)=\phi_{B}(t)-2 \pi f_{P N} t^{\prime} .
\end{aligned}
$$

Substituting (4) and (5) into (3), the observation difference can be simplified as

$$
\Theta(t)=2\left[\phi_{A}(t)-\phi_{B}(t)\right]
$$

Since the satellite clock and the PN code of the transmitter are driven by the same OSC, the time difference between two satellites can be derived from the PN code phase difference of the transmitter, and thus the time difference $\Delta T_{A B}$ is

$$
\Delta T_{A B}=\frac{\phi_{A}(t)-\phi_{B}(t)}{2 \pi f_{P N}}=\frac{\Theta(t)}{4 \pi f_{P N}} .
$$

\subsection{Full digital clock generation model}

The full digital clock generator (FDCG) has the capability to generate the adjustable frequency $[32,33]$. The dotted lines in Fig. 1 indicate the frequency connection relationship. The OSC of the follower provides the original reference frequency $f_{O S C}$ for the frequency multiplier (FM), so that the FDCG will be driven by a high-frequency clock $f_{m}$. The $f_{m}$ should be as large as possible since the FDCG will regenerate the reference frequency $f_{O S C}^{\prime}$ $\left(f_{O S C}^{\prime} \approx f_{O S C}\right)$ for the transponder and the clock of the satellite. The $f_{O S C}^{\prime}$ is determined by the bias frequency $\Delta f$, which is provided by the time difference controller (TDC).

The model of FDCG is shown in Fig. 2. The $f_{O S C}$ represents the nominal frequency of the oscillator. The direct output frequency of numerically controlled oscillator (NCO) must have large frequency spurs which are caused by the periodically $\mathrm{NCO}$ phase overflow in time domain. Consequently, the spur reduction technique (dither code) [34] and charge-pump phase locked loop (PLL) [35] are used for the regeneration of a high-quality reference frequency.

The dither code is a random number generator, which disrupts the periodicity of the NCO phase overflow, so the power of frequency spurs will be whitened. Since the PLL bandwidth is very small, most noise will be suppressed, 
thus the charge-pump PLL can be used to extract the highquality single tone analog signal from the digital output frequency of NCO which is contaminated by phase noise $[32,35]$. Therefore, the adjustable frequency $f_{O S C}^{\prime}$ can be used as a reference frequency for the system.

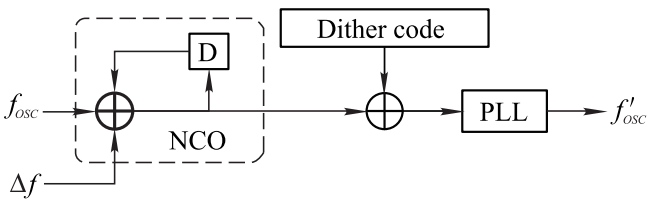

Fig. 2 FDCG model

\subsection{Time sync system model}

The model of time sync is illustrated in Fig. 3. The inputs of the TDMS equivalent model are $\theta_{c l k_{-} B}$ and $\theta_{c l k_{-} A}$, which represents the satellite clock phase of the leader and the follower, respectively. The time difference, denoted as $\Delta T$, is derived from clock phase difference by scaling $2 \pi f_{0}$, where $f_{0}$ is the nominal frequency of reference clock. An ideal first-order proportional-integral (PI) controller is adopted as the TDC. $K_{P}, K_{I} T_{u}$ are the coefficients of the proportional path and the integral path, respectively. Since the charge-pump PLL and dither code do not affect the value of output frequency of NCO, the FDCG is an NCO essentially. Consequently, FDCG is equivalently modeled as an accumulator with a coefficient of $2 \pi T_{u} . T_{u}$ is the update time interval of "time control loop", which is also equating to the frequency adjustment interval. There are carrier loop and delay lock loop (DLL) in the receiver of the DSSS baseband of the transponder. These loops will experience a step response at the bias reference frequency updating moment, since the system reference frequency is slightly changed by TDCS. In order to avoid loop coupling and ensure the stability of the system, the transient response of the loop in baseband should be less than $T_{u}$. According to the empirical relationship between bandwidth and response time [36], the update time of time control loop satisfies

$$
T_{u} \geqslant \frac{4}{B_{L_{-} \min }}
$$

where $B_{L_{-} \text {min }}$ is the minimum bandwidth in the baseband. Since TDMS is based on the PN code, the time difference measurement accuracy is depending on the loop bandwidth of DLL, which means that the minimum bandwidth appears in DLL.

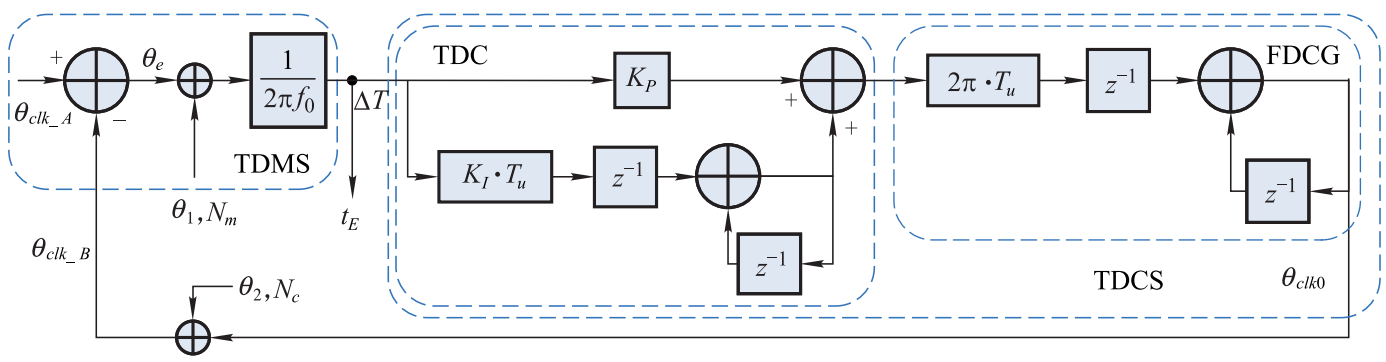

Fig. 3 Time sync model

Referring to Fig. 3, the system open-loop transfer function $G(z)$ can be obtained when setting input errors $\theta_{1}, \theta_{2}$ to be zero.

$$
\begin{aligned}
G(z)= & \frac{1}{2 \pi f_{0}}\left(K_{P}+\frac{K_{I} T_{u} z^{-1}}{1-z^{1}}\right) \frac{2 \pi T_{u} z^{-1}}{1-z^{-1}}= \\
& \frac{T_{u}}{f_{0}} \frac{K_{P} z-K_{P}+K_{I} T_{u}}{(z-1)^{2}}
\end{aligned}
$$

The closed-loop transfer function can be derived from $G(z)$ :

$$
\begin{gathered}
H(z)=\frac{G(z)}{1+G(z)}= \\
\frac{K_{P} T_{u} z-K_{P} T_{u}+K_{I} T_{u}^{2}}{f_{0} z^{2}+\left(K_{P} T_{u}-2 f_{0}\right) z+K}
\end{gathered}
$$

where $K=K_{I} T_{u}^{2}-K_{P} T_{u}+f_{0}$. There are three coefficients in (10). $K_{P}$ and $K_{I}$ are related to the natural angular frequency $\omega_{T O P}$ and damping coefficient $\xi_{T O P}$ of the time control loop, in this work, we use $\omega_{T O P}=0.1 \pi / T_{u}$, and $\xi_{T O P}=0.707$. The most important coefficient is $T_{u}$, which will affect the time sync accuracy and this will be discussed in the next sub-section.

\subsection{Time sync accuracy}

Time sync accuracy $\sigma_{\Delta T}$ is judged by the standard deviation (STD) of measured results (at node $t_{E}$ in Fig. 3) in the closed loop state. The sources of error in this model are the phase jitters due to oscillator drifting error $\sigma_{O}$, time difference measurement error $\sigma_{M}$, and clock compensation error $\sigma_{C}$.

\subsubsection{Oscillator drifting error}

The common measure of oscillator stability is the Allan Deviation (ADEV), $\sigma_{O S C}(\tau)$ [21], defined by 


$$
\sigma_{O S C}^{2}(\tau)=\frac{1}{2 N} \sum_{n=1}^{N} \frac{(f((n+1) \tau)-f(n \tau))^{2}}{f_{0}^{2}}
$$

where $f(n \tau)$ is the instantaneous frequency at time $n \cdot \tau$, and $f_{0}$ is used for normalizing. The ADEV represents the normalized frequency jitter between interval $\tau$, so the satellite clock jitter $\sigma_{c l k}$ caused by the oscillator can be expressed as

$$
\sigma_{c l k}=f_{0} \sigma_{O S C}(\tau)
$$

If the update time $T_{u}$ of the time control loop is very short, and equals $\tau$, the frequency jitter $\Delta f_{0}$ can be considered linearly. The phase jitter $\Delta \theta$ of satellite clock can be expressed as the integration of the frequency jitter.

$$
\begin{gathered}
\Delta \theta=2 \pi \int_{T_{0}}^{T_{0}+T_{u}} \Delta f_{0} \mathrm{~d} t= \\
\pi \Delta f_{0} T_{u}
\end{gathered}
$$

According to (12) and (13), the relationship between satellite clock phase jitter and ADEV can be written as

$$
\sigma_{\Delta \theta}=\pi \sigma_{c l k} T_{u}=\pi T_{u} f_{0} \sigma_{O S C}(\tau)
$$

where $\tau=T_{u}$. Reference [37] gives similar result. Due to the relationship between time difference and phase difference, the time sync error caused by oscillator drifting is supposed to be scaled by $\left(2 \pi f_{0}\right)^{-1}$ :

$$
\sigma_{O}=\frac{1}{2 \pi f_{0}} \cdot \sigma_{\Delta \theta}=\frac{1}{2} T_{u} \sigma_{O S C}(\tau) .
$$

The oscillator drifting error due to phase jitter is contributed by both the leader and the follower. In practice, the type of oscillators on these two satellites are the same, and hence the corrected oscillator drifting error can be expressed as

$$
\sigma_{\Delta T_{-} O}=\frac{\sqrt{2}}{2} T_{u} \sigma_{O S C}(\tau) .
$$

\subsubsection{Time difference measurement error and clock compensation error}

The time difference measurement error caused by thermal noise follows the Gaussian distribution. In this model, we assume that the clock compensation error also obeys the Gaussian distribution. Consequently, the equivalent single side band (SSB) noise power spectrum density of measurement error $N_{m}$ and compensation error $N_{c}$ can be defined as the ratio between noise and bandwidth:

$$
N_{m}=\frac{\sigma_{\theta M}^{2}}{\frac{1}{2} f_{u}}=8 \pi^{2} f_{0}^{2} \sigma_{M}^{2} T_{u}
$$

$$
N_{c}=\frac{\sigma_{\theta C}^{2}}{\frac{1}{2} f_{u}}=8 \pi^{2} f_{0}^{2} \sigma_{C}^{2} T_{u}
$$

where $f_{u}=1 / T_{u}, \sigma_{\theta M}$ and $\sigma_{\theta C}$ are the clock phase error derived from the corresponding time difference measurement error and clock compensation error respectively:

$$
\begin{gathered}
\sigma_{\theta M}=2 \pi f_{0} \sigma_{M} \\
\sigma_{\theta C}=2 \pi f_{0} \sigma_{C} .
\end{gathered}
$$

The measurement error emerges at the measuring process, and the compensation error enters the model at the feedback path. Therefore, the entrances of these errors are shown in Fig. 3, denoted as $\theta_{1}$ and $\theta_{2}$ respectively. The relationship between $\theta_{1}$ and $t_{E}$ indicates the effect of measurement error on time sync accuracy. Setting the irrelevant inputs to be zero except for $\theta_{1}$, the following relationships can be extracted from the model:

$$
\left\{\begin{array}{l}
t_{E}=\frac{\theta_{e}+\theta_{1}}{2 \pi f_{0}} \\
\theta_{c l k_{-} A}=\theta_{c l k 0} \\
\theta_{c l k 0}=t_{E}\left(K_{P}+\frac{K_{I} T_{u} z^{-1}}{1-z^{1}}\right) \frac{2 \pi T_{u} z^{-1}}{1-z^{-1}} \\
\theta_{e}=-\theta_{c l k_{-}} \\
H_{n 1}(z)=\frac{t_{E}}{\theta_{1}}
\end{array}\right.
$$

where $H_{n 1}(z)$ is the error transfer function between $\theta_{1}$ and $t_{E}, \theta_{c l k 0}$ is the original satellite clock phase before clock compensation. From the equation set above, we can obtain the expression of $H_{n 1}(z)$. The frequency response $H_{n 1}(\mathrm{j} \omega)$ can be given by setting $z=\mathrm{e}^{\mathrm{j} \omega}$, as shown in (22):

$$
H_{n 1}(\mathrm{j} \omega)=\frac{1}{2 \pi} \frac{\mathrm{e}^{2 \mathrm{j} \omega}-2 \mathrm{e}^{\mathrm{j} \omega}+1}{f_{0} \mathrm{e}^{2 \mathrm{j} \omega}+\left(K_{P} T_{u}-2 f_{0}\right) \mathrm{e}^{\mathrm{j} \omega}+K} .
$$

Similarly, the frequency response $H_{n 2}(\mathrm{j} w)$ between $\theta_{2}$ and $t_{E}$ can be given by the same method:

$$
H_{n 2}(\mathrm{j} \omega)=\frac{-1}{2 \pi} \frac{\mathrm{e}^{2 \mathrm{j} \omega}-2 \mathrm{e}^{\mathrm{j} \omega}+1}{f_{0} \mathrm{e}^{2 \mathrm{j} \omega}+\left(K_{P} T_{u}-2 f_{0}\right) \mathrm{e}^{\mathrm{j} \omega}+K} .
$$

Comparing (22) with (23), the only difference is that $H_{n 1}(\mathrm{j} \omega)$ differs by $\pi$ from $H_{n 2}(\mathrm{j} w)$ in phase response.

\subsubsection{Theoretical time sync accuracy and its optimization}

The output noise of this system can be determined by the input SSB noise power spectrum density and its frequency response.

$$
\sigma_{\Delta T_{-} M}=\sqrt{\int_{0}^{\frac{\pi}{T_{u}}} N_{m}\left|H_{n 1}(\mathrm{j} 2 \pi f)\right|^{2} \mathrm{~d} f}
$$




$$
\sigma_{\Delta T_{-} C}=\sqrt{\int_{0}^{\frac{\pi}{T_{u}}} N_{c}\left|H_{n 2}(\mathrm{j} 2 \pi f)\right|^{2} \mathrm{~d} f}
$$

In combination with the error caused by oscillator drifting, the theoretical time sync accuracy $\sigma_{\Delta T}$ is

$$
\begin{gathered}
\sigma_{\Delta T}=\sqrt{\sigma_{\Delta T_{-} M}^{2}+\sigma_{\Delta T_{-} C}^{2}+\sigma_{\Delta T_{-} O}^{2}}= \\
\sqrt{k_{M} \sigma_{M}^{2}+k_{C} \sigma_{C}^{2}+k_{O} \sigma_{O}^{2}}
\end{gathered}
$$

where $k_{M}, k_{C}$ and $k_{O}$ are the coefficients of time difference measurement error, clock compensation error, and oscillator drifting error respectively, which can be obtained by substituting (16), (17), (18), (24) and (25) into (26).

$$
\begin{gathered}
k_{M}=8 \pi^{2} f_{0}^{2} T_{u} \int_{0}^{\frac{\pi}{T_{u}}}\left|H_{n 1}(\mathrm{j} 2 \pi f)\right|^{2} \mathrm{~d} f \\
k_{C}=8 \pi^{2} f_{0}^{2} T_{u} \int_{0}^{\frac{\pi}{T_{u}}}\left|H_{n 2}(\mathrm{j} 2 \pi f)\right|^{2} \mathrm{~d} f \\
k_{O}=\frac{1}{2} T_{u}^{2}
\end{gathered}
$$

Now, we will look for the optimum time sync performance. The update time of time control loop is not determined yet, since it will influence time sync performance dramatically. According to [38], we can infer that the time difference measurement error $\sigma_{M}^{\prime}$ due to thermal noise can be expressed as

$$
\sigma_{M}^{\prime}=\frac{\pi f_{0}}{f_{P N}} \sqrt{\frac{B_{L_{-} D L L}}{2 \frac{C}{N_{0}} B_{f e} T_{c}}\left(1+\frac{1}{T_{c o h} C / N_{0}}\right)}
$$

where $B_{L_{-} D L L}$ is the bandwidth of DLL, $C / N_{0}$ is the carrier-noise ratio (CNR), $T_{c o h}$ is the correlation integration time, $B_{f e}$ represents the double-sided front-end bandwidth, $T_{c}$ is the chip period. The measurement error is caused by both the leader and the follower as well, it can be corrected as $\sigma_{M}=\sqrt{2} \sigma_{M}^{\prime}$. Taking the lower limit of the inequality as shown in (8), we have the relationship between $T_{u}$ and $B_{L_{-} D L L}$ :

$$
T_{u}=\frac{4}{B_{L_{-} \min }}=\frac{4}{B_{L_{-} D L L}} .
$$

Therefore, by comparing (16) with (30), we can find that the oscillator drifting error is inversely proportional to the bandwidth, while time difference measurement error is proportional to the bandwidth, which indicates that the optimum accuracy of time sync may be obtained by making the compromise between oscillator drifting error and measurement error. The theoretical time sync accuracy is shown in Fig. 4. Because the chip rate of the DSSS transponder is $5.115 \mathrm{MHz}$, and considering the noise and dynamic performance of the receiver, the parameters should be set as the followings: $B_{f e}=20 \mathrm{MHz}$, $T_{c}=0.196 \mu \mathrm{s}, T_{c o h}=50 \mu \mathrm{s}, \sigma_{C}=10 \mathrm{ps}$ [32] and $\sigma_{O S C}(\tau)=2.5 \mathrm{ppb}$.

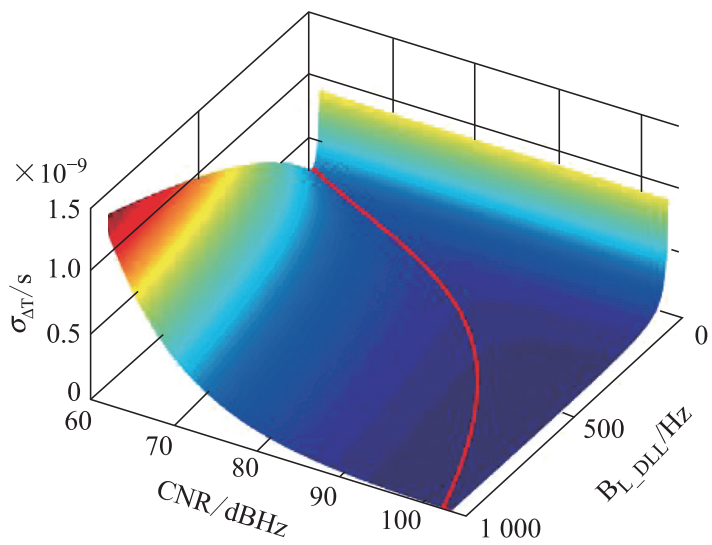

Fig. 4 Theoretical time sync accuracy

The surface in Fig. 4 is theoretical time difference accuracy with various combinations of the bandwidth and $\mathrm{CNR}$, and the highlight curve in that surface indicates the aggregation of optimum solutions. Regardless of the CNR condition, theoretical accuracy of time sync decreases when the bandwidth is small (particularly smaller than $50 \mathrm{~Hz}$ ), since the small bandwidth implies large $T_{u}$, which leads the sync accuracy to be dominated by oscillator drifting error. Although the effect of oscillator drifting error is greatly reduced as the bandwidth increases, the thermal noise, in turn, becomes the main contributor. Hence, CNR mostly determines time sync accuracy since the bandwidth is relatively large. However, consider the case with limited transmission rate, it takes time for the leader to send the observation $\Delta \phi_{B}(t)$ to the follower. Thus, the update time of the time control loop cannot be infinitely small. Taking all analyses above into account, we set the time control loop update time $T_{u}$ to be $0.02 \mathrm{~s}\left(B_{L_{-} D L L}=200 \mathrm{~Hz}\right)$ for the following simulation and experiment.

\section{Simulation results}

Simulations are carried out by the Matlab simulink toolkit to evaluate the functionality and performance of TDCS. The simulation model is shown in Fig. 5. The module "clockA" generates the clock phase of the leader; the follower's clock phase is generated by accumulating the frequency which is the sum of an initial reference frequency (df0) and the bias reference frequency determined by TDC; the switch is used for controlling the simulation state, we can observe the changing process from open loop state to closed loop state when the control signal is pulling up. The input of TDC is forced to zero in open loop state, which connects to time difference during closed loop state. 


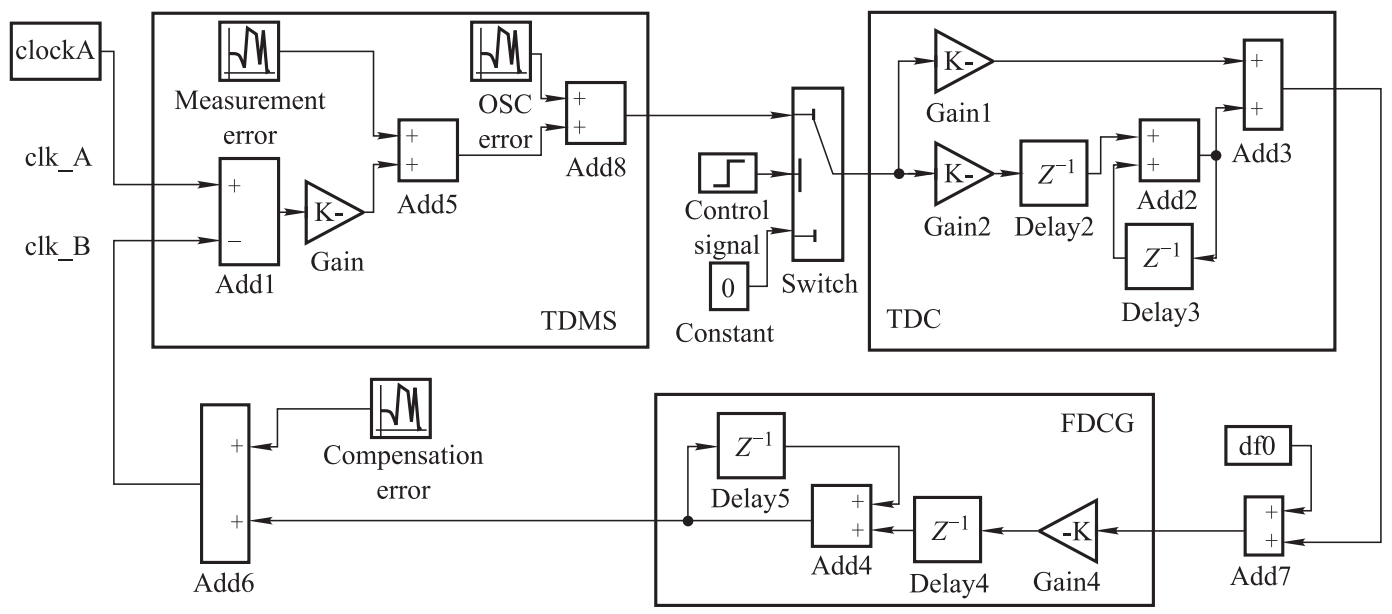

Fig. 5 TDCS simulation model

The measurement error, compensation error and oscillator error are inserted into simulation by the random number generators.

In this simulation, we assume that the initial bias reference frequency of the follower is $1 \mathrm{~Hz}$, and it will enter closed loop state after $8.6 \mathrm{~s}$. In order to observe the changing of time difference, the bias reference frequency of the leader is $0.5 \mathrm{~Hz}$ in the first $7.6 \mathrm{~s}$, and then it will be altered to $3 \mathrm{~Hz}$.

Fig. 6 illustrates the clock phase simulation results of the leader and the follower respectively. During the entire simulation, the clock phase of the leader is running independently, but that of the follower is running freely only in the open loop state. In the closed loop state, the clock phase of the follower will try to track the leader's clock phase. The coincidence of these two curves means that the time sync is achieved.

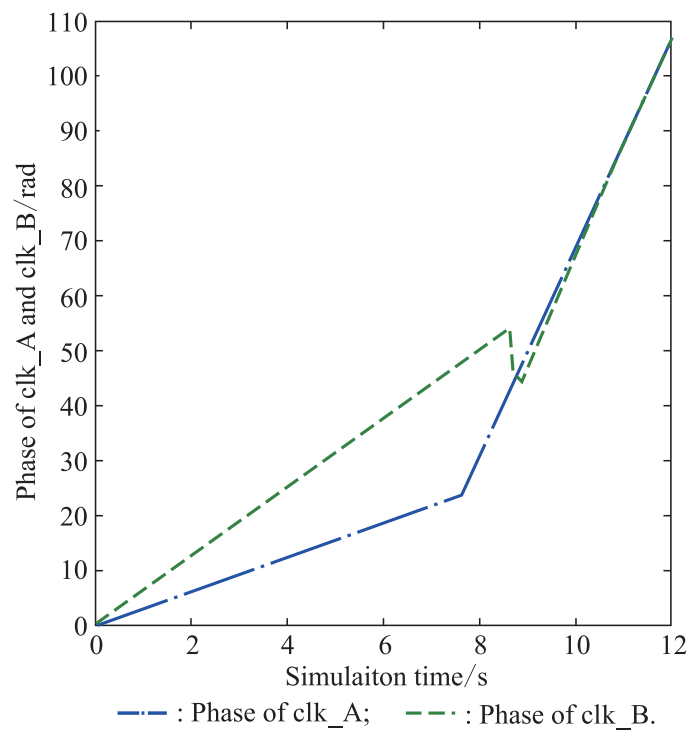

Fig. 6 Simulation results of clock phase
Fig. 7 gives the corresponding changes of time difference and bias reference frequency of the follower. Time difference is zero at the beginning of the simulation, but it is growing negatively with the rate of $-0.5 \mathrm{~Hz}$ before $7.6 \mathrm{~s}$, which meets the preset condition that the reference frequency of the follower is $0.5 \mathrm{~Hz}$ higher than that of the leader. It can also be verified by the slope of time difference:

$$
\Delta f=\frac{T d\left(t_{1}\right)-T d\left(t_{2}\right)}{t_{1}-t_{2}} \cdot f_{0}
$$

where $\Delta f$ represents instantaneous frequency difference, and $T d\left(t_{1}\right)$ is time difference at moment $t_{1}$. Time difference is changing with the rate of $2 \mathrm{~Hz}$ from $7.6 \mathrm{~s}$ to $8.6 \mathrm{~s}$, since the reference frequencies of the leader and the follower become $3 \mathrm{~Hz}$ and $1 \mathrm{~Hz}$ respectively. Then the loop is closed at $8.6 \mathrm{~s}$, after experiencing a dynamic adjustment process, the time difference and the reference frequency of the follower converge to approximately $0 \mathrm{~s}$ and $3 \mathrm{~Hz}$ finally.
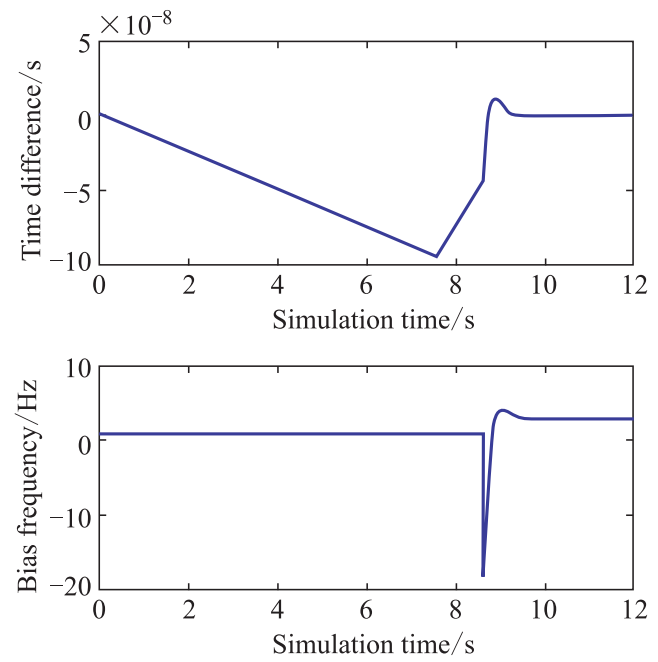

Fig. 7 Simulation results of time difference and bias reference frequency of the follower 
It is worthy of note that a frequency overshoot appears, and it may be harmful to the system. If a large frequency overshoot is applied, the tracking loop of receiver unlocks, skips cycles for a while, and then may lock up once again [39]. It may disable the time difference measurement system, resulting in the failure of time sync. Therefore, the frequency overshoot cannot exceed the DLL fast capture bandwidth [40]:

$$
\Omega=2 \xi_{D L L} \omega_{D L L}
$$

where $\Omega$ represents fast capture bandwidth, $\omega_{D L L}$ and $\xi_{D L L}$ are the nature angular frequency and damping coefficient of DLL. In this work, $\xi_{D L L}=0.707, \omega_{D L L} \approx$ $377 \mathrm{~Hz}$, and $\Omega=533 \mathrm{~Hz}$. Besides, the frequency overshoot is linearly related to the time difference. Therefore, we can derive the safety initial time difference $\left(t d_{i n i}\right)$ for entering closed loop state, below which the DLL does not unlock.

$$
t d_{i n i}=\alpha \frac{f_{0}}{f_{P N}} \Omega=2 \alpha \frac{f_{0}}{f_{P N}} \xi_{D L L} \omega_{D L L}
$$

where $\alpha$ is the scale factor, which can be obtained by several groups of simulation. In this case $\alpha=2.22 \times$ $10^{-9} \mathrm{~s} / \mathrm{Hz}$, so that $t d_{i n i}=9.27 \mu \mathrm{s}$.

The performance of time sync is evaluated by the STD of time difference results. In order to compare the theoretical and experimental result, we set $\sigma_{O S C}(\tau)=2.5$, $\sigma_{t_{-} c}=10 \mathrm{ps}$, which is similar to the experimental condition. The only independent variable is CNR which affects the measurement error. The simulation results are shown in Table 1, and the theoretical result is computed by (26).

Table 1 Simulation results and analytical results

\begin{tabular}{ccc}
\hline Simulation parameters & Simulation results & Theoretical result \\
\hline $\mathrm{CNR} / \mathrm{dBHz}$ & $\sigma_{\Delta T}^{\prime} / \mathrm{ps}$ & $\sigma_{\Delta T} / \mathrm{ps}$ \\
\hline 95 & 47.79 & 39.96 \\
90 & 58.08 & 45.39 \\
85 & 76.30 & 59.28 \\
80 & 109.0 & 90.07 \\
75 & 167.6 & 150.6 \\
70 & 272.2 & 262.2 \\
65 & 459.1 & 446.1 \\
\hline
\end{tabular}

\section{System demonstration}

In order to verify the system performance predicted by foregoing analysis and simulation, the experiment of time sync has been built with two S band transponders. Since the software defined radio (SDR) has the functional flexibility [41], the transponder utilizes a combination of field programmable gate array (FPGA) to accomplish the function of DSSS baseband and time sync. Fast Fourier transform is adopted by the DSSS baseband to capture the phase of the PN code and the Doppler frequency simultaneously [42]. The transponder applied with low power FPGA consumes only $4 \mathrm{~W}$ with the transmission power of $25 \mathrm{dBmW}$ and weighs less than $100 \mathrm{~g}$.

As the test bench shown in Fig. 8, the entire system is built with radio frequency cables to avoid the errors caused by antenna system. A pair of attenuators is inserted close to each transmission port to simulate the signal attenuation caused by free space loss. The communication between $\mathrm{PC}$ and the follower is realized through a serial port, from which we can obtain time difference measurement results, and to which we can send instructions. The instruction is used to set the working state of the follower, so that we can clearly observe the changing process between open loop state and closed loop state.

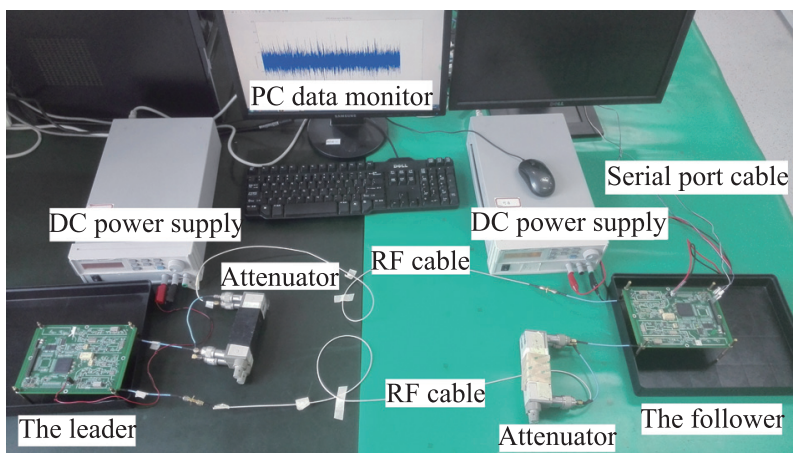

Fig. 8 Test bench

The workflow of the system is introduced as shown in Fig. 9. The seven steps of system working process not only prevent the frequency overshoot but also shorten the elapsing time before achieving time sync.

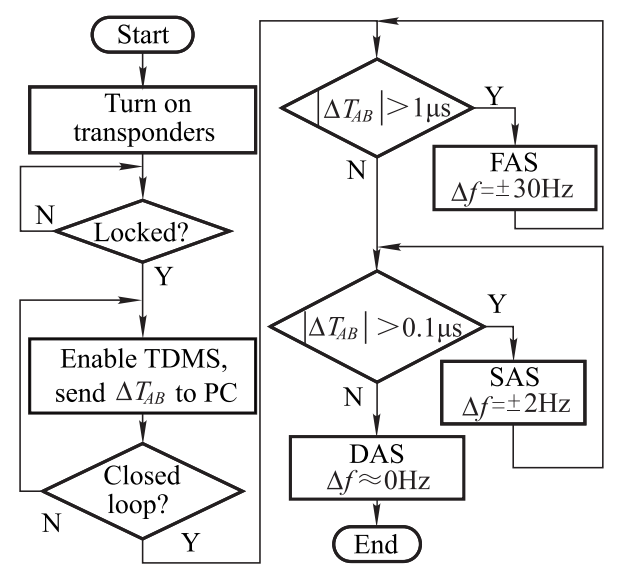

Fig. 9 Workflow of time sync system

Step 1 Turn on the leader and the follower, respectively.

Step 2 Waiting for a while until the uplink and downlink signal to be locked. 
Step 3 The follower enables the TDMS and sends the time difference $\Delta T_{A B}$ to PC.

Step 4 If the "closed loop" instruction is received, the follower enables TDCS. Otherwise maintain the original state.

Step 5 If the time difference is larger than $1 \mu \mathrm{s}$, then go to fast adjust state (FAS), during which the bias frequency is $\pm 30 \mathrm{~Hz}$. Otherwise, move to next step.

Step 6 If the time difference is larger than $0.1 \mu \mathrm{s}$, then go to slow adjust state (SAS), during which the bias frequency is $\pm 2 \mathrm{~Hz}$. Otherwise, move to dynamic adjust state (DAS).

Step 7 Once entering DAS, the bias frequency is dynamically adjusted by TDC referring to the current time difference provided by TDMS, thus the time difference can be kept around zero.

Fig. 10 shows the experiment result of time difference and frequency difference (derived by (32)). From $0 \mathrm{~s}$ to $23 \mathrm{~s}$, the initial frequency difference between the independent oscillators is about $15 \mathrm{~Hz}$. The turning point of time difference indicates that the follower receives an instruction and enters closed loop state. Before DAS, the measured bias frequencies are about $-30 \mathrm{~Hz}$ and $-2 \mathrm{~Hz}$ in FAS and SAS, respectively, which meets the preset condition.
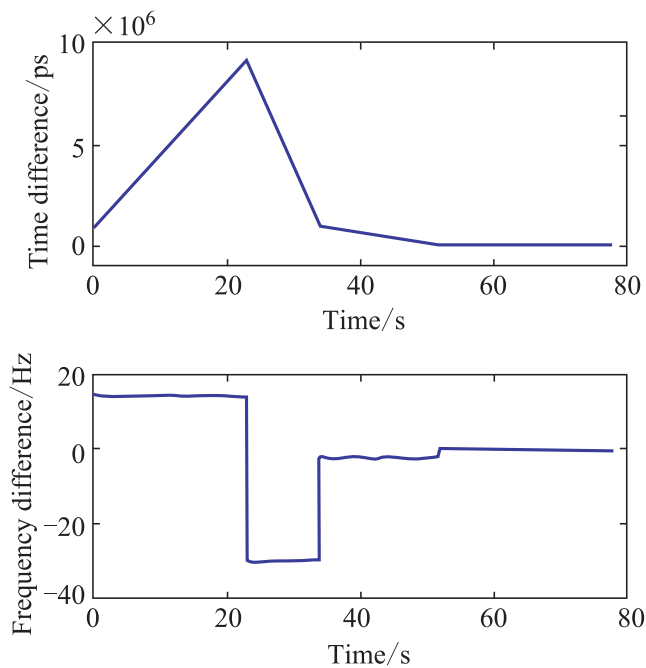

Fig. 10 Time sync process

Experiments are carried out under various CNRs to testify the long-term stability of the system as shown in Fig. 11(a) to Fig. 11(e). Compared with Fig. 10, the time difference is successfully reduced and maintained around zero during the 50 minutes experiment, and it will keep stable along with the experiment going on. Table 2 gives the STD of time difference and frequency difference, which are calculated from the valid data during DAS, the best time sync accuracy reaches about 102 ps under the condition of $95 \mathrm{dBHz}$, and the corresponding frequency accuracy is about $0.36 \mathrm{~Hz}$.

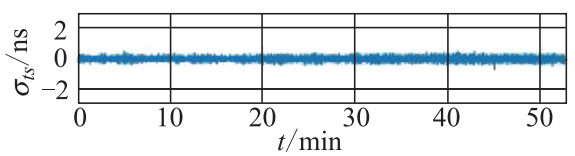

(a) $\mathrm{CNR}=92 \mathrm{dBHz}, \mathrm{STD}=101.68 \mathrm{ps}$

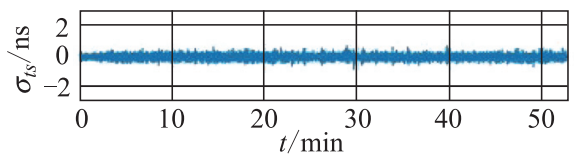

(b) $\mathrm{CNR}=85 \mathrm{dBHz}, \mathrm{STD}=137.9011 \mathrm{ps}$

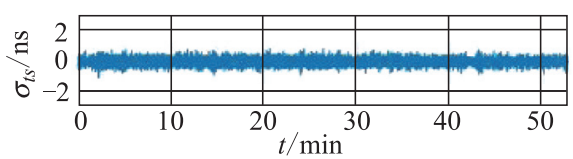

(c) $\mathrm{CNR}=80 \mathrm{dBHz}, \mathrm{STD}=186.06 \mathrm{ps}$

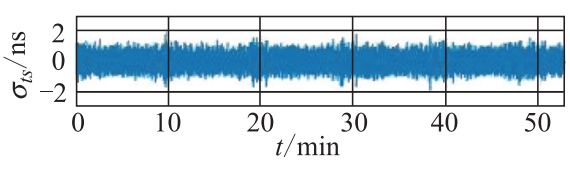

(d) $\mathrm{CNR}=75 \mathrm{dBHz}, \mathrm{STD}=305.9401 \mathrm{ps}$

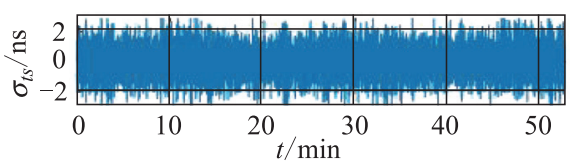

(e) $\mathrm{CNR}=65 \mathrm{dBHz}, \mathrm{STD}=770.7202 \mathrm{ps}$

Fig. 11 Experimental results in DAS

Table 2 Time difference results in lab

\begin{tabular}{ccc}
\hline $\mathrm{CNR} / \mathrm{dBHz}$ & STD of time difference/ps & STD of frequency/Hz \\
\hline 95 & 101.68 & 0.3619 \\
90 & 114.20 & 0.4067 \\
85 & 137.90 & 0.5531 \\
80 & 186.06 & 0.8391 \\
75 & 305.94 & 1.4509 \\
70 & 487.53 & 2.3243 \\
65 & 770.72 & 3.5434 \\
\hline
\end{tabular}

\section{Conclusions}

In this paper, we propose the TDCS, which can establish a unified time benchmark in a mcro-satellite cluster by synchronizing the clock of the follower to that of the leader. TDCS mainly consists of a TDC and an FDCG. The TDC could dynamically compute the bias reference frequency to compensate for time difference. The FDCG is driven by the OSC but generates the adjustable reference frequency for the follower under the control of TDC. Consequently, time difference can be reduced to and kept around zero as long as this system is properly working. The whole system does not require atomic frequency standards or dedicated 
ground stations for the maintenance of time synchronization, and it is also free of post-process, which is beneficial to minimize human intervention and realize autonomous operation of micro-satellite cluster. Finally, the experimental result shows that the standard deviation of time synchronization is about 102 ps when CNR is $95 \mathrm{dBHz}$, and the standard deviation of the corresponding frequency is approximately $0.36 \mathrm{~Hz}$.

\section{References}

[1] FROST C, AGASID E. Small spacecraft technology state of the art. NASA Technical Report TP-2014-216648/REV1, NASA Ames Research Center, 2014.

[2] MATHIEU C, WEIGEL A L. Assessing the flexibility provided by fractionated spacecraft. Proc. of AIAA SPACE Forum, 2005, 1: 1- 12 .

[3] POGHOSYAN A, GOLKAR A. CubeSat evolution: analyzing CubeSat capabilities for conducting science missions. Progress in Aerospace Sciences, 2017, 88: 59-83.

[4] GILL E, SUNDARAMOORTHY P, BOUWMEESTER J, et al. Formation flying within a constellation of nano-satellites: the QB50 mission. Acta Astronautica, 2013, 82(1): 110-117.

[5] MCCORMICK D, BARRETT B, BURNSIDECLAPP M. Analyzing fractionated satellite architectures using RAFTIMATE: a Boeing tool for value-centric design. Proc. of AIAA SPACE Conference \& Exposition, 2013, 6767: 1 -6.

[6] D'ERRICO M. Distributed space missions for earth system monitoring. New York: Microcosm Press and Springer, 2013.

[7] SHAW G B, MILLER D W, HASTINGS D E. Generalized characteristics of communication, sensing, and navigation satellite systems. Journal of Spacecraft and Rockets, 2000, 37(6): $801-811$.

[8] CELANDRONI N, FERRO E, GOTTA A, et al. On the applicability of reliable transport protocols in satellite delay tolerant and disruptive networks. International Journal of Satellite Communications \& Networking, 2014, 32(2): 141-161.

[9] WANG C, TANG J H, CHENG X H, et al. Distributed cooperative ask planning algorithm for multiple satellites in delayed communication environment. Journal of Systems Engineering and Electronics, 2016, 27(3): 619-633.

[10] RADHAKRISHNAN R, EDMONSON W W, et al. Optimal multiple access protocol for inter-satellite communication in small satellite system. Proc. of 4s Small Satellite Systems and Services Symposium, 2014: 1-15.

[11] TAPLEY B D, BETTADPUR S, WATKINS M, et al. The gravity recovery and climate experiment: mission overview and early results. Geophysical Research Letters, 2004, 31(9): 10.1029/2004GL019779.

[12] BAGUIO M, TEACHERS G M. Amazing GRACE: NASA's gravity recovery and climate experiment. Proc. of Lunar and Planetary Science Conference, 2008: 18-22.

[13] ORR N G, EYER J K, LAROUCHE B P, et al. Precision formation flight: The Can X-4 and Can X -5 Dual Nanosatellite Mission. ESA Special Publication, 2008: 1-10.

[14] LANDGRAF M, MESTREAU-GARREAU A. Formation flying and mission design for Proba-3. Acta Astronautica, 2013, 82(1): $137-145$.

[15] LlORENTE J S, AGENJO A, CARRASCOSA C, et al. PROBA-3: Precise formation flying demonstration mission. Acta Astronautica, 2013, 82(1): 38-46.

[16] GILL E, MONTENBRUCK O, D’AMICO S. Autonomous formation flying for the PRISMA mission. Journal of Spacecraft and Rockets, 2007, 44(3): 671-681.
[17] ARDAENS J S, KAHLE R, SCHULZE D. In-flight performance validation of the TanDEM-X autonomous formation flying system. International Journal of Space Science and Engineering, 2014, 2(2): $157-170$.

[18] PITZ W, MILLER D. The TerraSAR-X satellite. IEEE Trans. on Geoscience and Remote Sensing, 2010, 48(2): 615-622.

[19] ZHANG H, GURFIL P. Distributed control for satellite cluster flight under different communication topologies. Journal of Guidance Control \& Dynamics, 2015, 39(3): 1-11.

[20] RADHAKRISHNAN R, EDMONSON W W, AFGHAH F, et al. Survey of inter-satellite communication for small satellite systems: physical layer to network layer view. IEEE Communications Surveys \& Tutorials, 2016, 18(4): $2442-2473$.

[21] KAPLAN E D, HEGARTY C J. Understanding GPS: Principles and Applications. 2nd ed. London: Artech House Inc., 2006.

[22] TAYLOR J, BARNES E. GPS current signal-in-space navigation performance. Proc. of the National Technical Meeting of the Institute of Navigation, 2005: 385-393.

[23] BERTIGER W, BAR-SEVER Y, DESAI S, et al. GRACE: millimeters and microns in orbit. Gdgps Net, 2002: 2022 -2029.

[24] PARK R, KONOPLIV A, YUAN D N, et al. High-resolution lunar gravity from the gravity recovery and interior laboratory mission. Proc. of the 23rd AAS/AIAA Spaceflight Mechanics Meeting, paper AAS. 13-272.

[25] KLIPSTEIN W M, ARNOLD B W, ENZER D G, et al. The lunar gravity ranging system for the Gravity Recovery and Interior Laboratory (GRAIL) mission. Space Science Reviews, 2013, 178(1): $57-76$.

[26] DUNN C, BERTIGER W, FRANKLIN G, et al. The Instrument on NASA's GRACE mission: augmentation of GPS to achieve unprecedented gravity field measurements. Proc. of the International Technical Meeting of the Satellite Division of the Institute of Navigation, 2002: 724-730.

[27] ASMAR S W, KONOPLIV A S, WATKINS M M, et al. The scientific measurement system of the Gravity Recovery and Interior Laboratory (GRAIL) mission. Space Science Reviews, 2013, 178(1): 25-55.

[28] HUANG F, LU X, WU H, et al. Algorithm of intersatellite dynamic two-way time transfer based on GEO satellite. Proc. of the IEEE International Frequency Control Symposium Joint with the European Frequency and Time Forum, 2009: $688-$ 691.

[29] HUANG Y J, TSENG W H, LIN S Y, et al. Introduction of software-defined receivers in two-way satellite time and frequency transfer. Proc. of the IEEE International Frequency Control Symposium, 2016: 1-26.

[30] YAO J, SKAKUN I, JIANG Z, et al. Comparison of two continuous GPS carrier-phase time transfer techniques. Proc. of the IEEE Frequency Control Symposium \& the European Frequency and Time Forum, 2015: 655-661.

[31] DACH R, HUGENTOBLER U, SCHILDKNECHT T, et al. Precise continuous time and frequency transfer using GPS carrier phase. Proc. of the IEEE International Frequency Control Symposium and Exposition, 2006: 329-336.

[32] XU P P, ZHANG C J, LOU Y N, et al. FPGA-based all-digital clock generation method. Journal of Zhejiang University (Engineering Science), 2017 (12): 2341 -2347. (in Chinese)

[33] LOU Y N, JIN Z H, ZHANG C J. A method of full digital clock generation with adjustable frequency and phase. Applied Mechanics \& Materials, 2014, 599/601: 703-706.

[34] VANKKA J, HALONEN K. Spur reduction techniques in sine output direct digital synthesizer. Digital Synthesizers and Transmitters for Software Radio, 2005: 113-137.

[35] XU X, LIU H, TAN W. Parameters design of $1.25 \mathrm{GHz}$ low jit- 
ter charge pump PLL. Proc. of the IEEE International Conference on Electric Information and Control Engineering, 2011: $3418-3421$.

[36] LOU Y N, JIN Z H, ZHANG C J. A method of full digital clock generation with adjustable frequency and phase. Journal of Systems Engineering and Electronics, 2014, 25(6): $949-$ 958.

[37] GARDNER F M. Phaselock Techniques. 3rd ed. New York: Wiley, 2005.

[38] BETZ J, KOLODZIEJSKI K. Extended theory of early-late code tracking for a band limited GPS receiver. Journal of the Institute of Navigation, 2000, 47(3): 211-226.

[39] HUQUE A, STENSBY J. An analytical approximation for the pull-out frequency of a PLL employing a sinusoidal phase detector. ETRI Journal, 2013, 35(2): 218-225.

[40] STENSBY J. An approximation of the pull-out frequency parameter in a second-order PLL. Proc. of the 38th Southeastern Symposium on System Theory, 2006: 75 - 79.

[41] MAHESHWARAPPA M R, BRIDGES C P. Software defined radios for small satellites. Proc. of the NASA/ESA Conference on Adaptive Hardware and Systems (AHS), 2014: 172 - 179.

[42] ZHANG H, XU L, JIAN Y, et al. A 2-step GPS carrier tracking loop for urban vehicle applications. Journal of Systems Engineering and Electronics, 2017, 28(5): 817-826.

\section{Biographies}

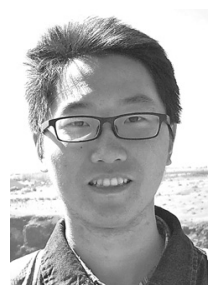

XU Jiuling was born in 1990. He received his B.E. degree in communication engineering from Zhejiang University of Technology. He is a member of the Micro-Satellite Research Center, Zhejiang University. His research interests include micro transponder, inter-satellite ranging and time synchronization.

E-mail: xu90@zju.edu.cn

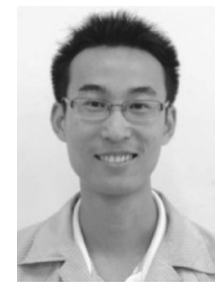

ZHANG Chaojie was born in 1982. He received his B.E and Ph.D. degrees from Zhejiang University in 2004 and 2009, respectively. Now he is an associate researcher of Zhejiang University. He has joined in the Micro-Satellite Research Center since he was a graduate student. His research interests include micro-satellite and software defined radio technologies.

E-mail: zhangcj@zju.edu.cn

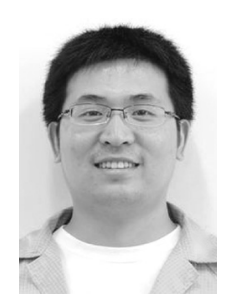

WANG Chunhui was born in 1983. He received his B.E and Ph.D. degrees from Zhejiang University in 2004 and 2009, respectively. Now he is an associate researcher of Zhejiang University. He has joined in the Micro-Satellite Research Center since he was a graduate student. His research interests include analog transponder and high accuracy intersatellite ranging system.

E-mail: hytgwch@zju.edu.cn

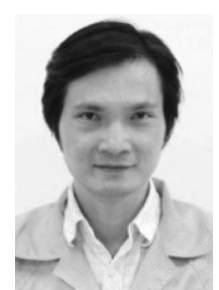

JIN Xiaojun was born in 1977. He received his B.E, M.E. and Ph.D. degrees from Zhejiang University in 2001, 2004 and 2007, respectively. He joined the faculty of Zhejiang University in 2009, and has been an associate professor since 2010. His research interests include micro satellite transponder technology, relative ranging and navigation of micro-satellite formation flying. E-mail: axemaster@zju.edu.cn 